\title{
Fabricating Silver Nanoparticles on Thin Silicon Nanowalls for Highly Sensitive Surface-Enhanced Raman Scattering
}

\author{
Shu Ning Wen and Jiann Shieh* \\ Department of Materials Science and Engineering, National United University, \\ 1, Lienda, Miaoli 36003, Taiwan
}

\begin{abstract}
Metal nanoparticles with nanoscale spacing are promising materials for the detection of single molecules through surface-enhanced Raman scattering. To increase the sensitivity of nanoparticles through the use of a nanoscale substrate, we fabricated various Ag NP-decorated silicon nanowalls for the Raman spectroscopic detection of rhodamine 6G (R6G). The sensitivity of detection was affected by the nanowall depth and was influenced by several parameters: the $\mathrm{AgNO}_{3}$ concentration for metal-assisted etching, the $\mathrm{HF} / \mathrm{H}_{2} \mathrm{O}_{2}$ etching time for nanowall formation, and the Ag evaporation time for nanoparticle growth. For an approximately 400-nm-deep nanowall substrate having the optimal surface filling ratio and etching depth, we obtained an ultrahigh enhancement factor of $1.1 \times 10^{9}$ for the detection of R6G at a concentration of $10^{-11} \mathrm{M}$. [doi:10.2320/matertrans.M2014259]
\end{abstract}

(Received July 14, 2014; Accepted September 11, 2014; Published October 18, 2014)

Keywords: silver, surface-enhanced Raman scattering, nanowall, metal-assisted chemical etching

\section{Introduction}

Surface-enhanced Raman scattering (SERS) is an excellent method for detecting molecules at low concentration. ${ }^{1)}$ As a result of surface chemical enhancement and electromagnetic effects associated with surface plasmon resonance, especially from "hot spots" between nanoparticles (NPs), nanostructured metal-assisted SERS can increase the Raman scattering cross-sections and, thereby, improve sensitivity. ${ }^{2}$ Many methods, including chemical reduction, electrochemical deposition, and vapor deposition, ${ }^{3)}$ are available for the preparation of metal NPs. On two-dimensional (2D) substrates, the Raman sensitivity can be enhanced by greater than $10^{5}$ through the assistance of deposited NPs. ${ }^{4)}$ To improve the sensitivity further, several three-dimensional (3D) nanostructures, including nanotips, ${ }^{5,6}$ nanotubes, ${ }^{7)}$ nanowires, ${ }^{8-10)}$ nanowalls, ${ }^{11)}$ nanoforests, ${ }^{12)}$ and nanocanals, ${ }^{13)}$ have been investigated in conjunction with various nanofabrication processes. Among them, metal-assisted chemical etching (MACE) has attracted much attention because it is a simple and inexpensive process that can produce structures over large areas. ${ }^{14)}$ Unfortunately, the etching rate is so high that thick nanostructures (several tens of micrometers) are usually formed, thereby limiting the SERS applications to microdevices. In addition, only limited investigations have been made into the effects of microstructures on the SERS sensitivity. In this present study, we systematically investigated the preparation of thin-layer nanostructures - varying the $\mathrm{AgNO}_{3}$ concentration, the $\mathrm{HF}$ / $\mathrm{H}_{2} \mathrm{O}_{2}$ etching time, and the Ag deposition time-to optimize the sensitivity of SERS toward rhodamine 6G (R6G). Using thermal evaporation to decorate Ag NPs on Si nanowalls, we found that there exists an optimal profile to obtain the highest SERS sensitivity, confirmed through microscopy images of the silicon nanowall structures. This optimal profile, characterized by a silicon nanowall depth of only $400 \mathrm{~nm}$, can be used to detect low concentrations of R6G with ultrahigh sensitivity [enhancement factor $(E F)$ : $>10^{11}$ ].

*Corresponding author, E-mail: jshieh@ nuu.edu.tw

\section{Experimental Procedure}

(100) Silicon wafers having a resistance of 1.5-100 ohm-cm (test grade) were washed sequentially with acetone, ethanol, and DI water and then immersed in a solution containing $\mathrm{H}_{2} \mathrm{SO}_{4}(98$ mass $\%)$ and $\mathrm{H}_{2} \mathrm{O}_{2}$ (30 mass \%), at a volume ratio of $4: 1$, at $90^{\circ} \mathrm{C}$ for $15 \mathrm{~min}$ to remove any organic impurities and also to form a thin oxide layer on the surface. A buffer containing $\mathrm{HF}$ ( 5 mass $\%$ ) was then used to remove the oxide and to create a H-terminated surface. The samples were then immersed in a solution containing $\mathrm{HF}(5 \mathrm{M})$ and $\mathrm{AgNO}_{3}$ $(0.001-0.06 \mathrm{M})$ to form Ag NPs on the wafer surface. The unadsorbed Ag NPs that formed in solution were washed away with DI water to slow down the etching rate and to improve the uniformity in height. ${ }^{15)}$ An etching solution comprising $\mathrm{HF}(5 \mathrm{M})$ and $\mathrm{H}_{2} \mathrm{O}_{2}(0.1 \mathrm{M})$ was applied for $10-1800 \mathrm{~s}$ to etch down the silicon to form the silicon nanowalls with assistance from the Ag NPs. The residual Ag NPs were removed using a $\mathrm{HNO}_{3}$ solution and then the samples were washed with DI water and dried under a flow of $\mathrm{N}_{2}$.

For SERS measurements, Ag NPs were deposited on the nanowalls through thermal evaporation (KD-thermal system) for 30-300 s. Nanoparticles can be immobilized on $\mathrm{Si}$ substrates through the interactions between $\mathrm{Ag}$ atoms and Si wafer during deposition. ${ }^{16}$ The samples were placed in $0.1 \mathrm{M} \mathrm{KCl}$ for $60 \mathrm{~min}$ to activate the surface for Raman measurements. They were immersed in a 100-ml R6G solution (from $10^{-7}$ to $10^{-11} \mathrm{M}$ ) for $1 \mathrm{~h}$ and then dried under a flow of $\mathrm{N}_{2}$ prior to SERS measurements.

The morphologies of the samples were characterized through scanning electron microscopy (SEM) using a JEOL-6700F microscope. SERS measurements were performed using a micro-Raman system (Jobin Yvon-Labram HR) with excitation of light (wavelength: $532 \mathrm{~nm}$ ) from a $\mathrm{Nd}$ :YAG laser. The spot size was focused to approximately $1 \mu \mathrm{m}$; the objective lens was $100 \times$; the power was $3 \mathrm{~mW}$; and the accumulating time was $1 \mathrm{~s}$. 


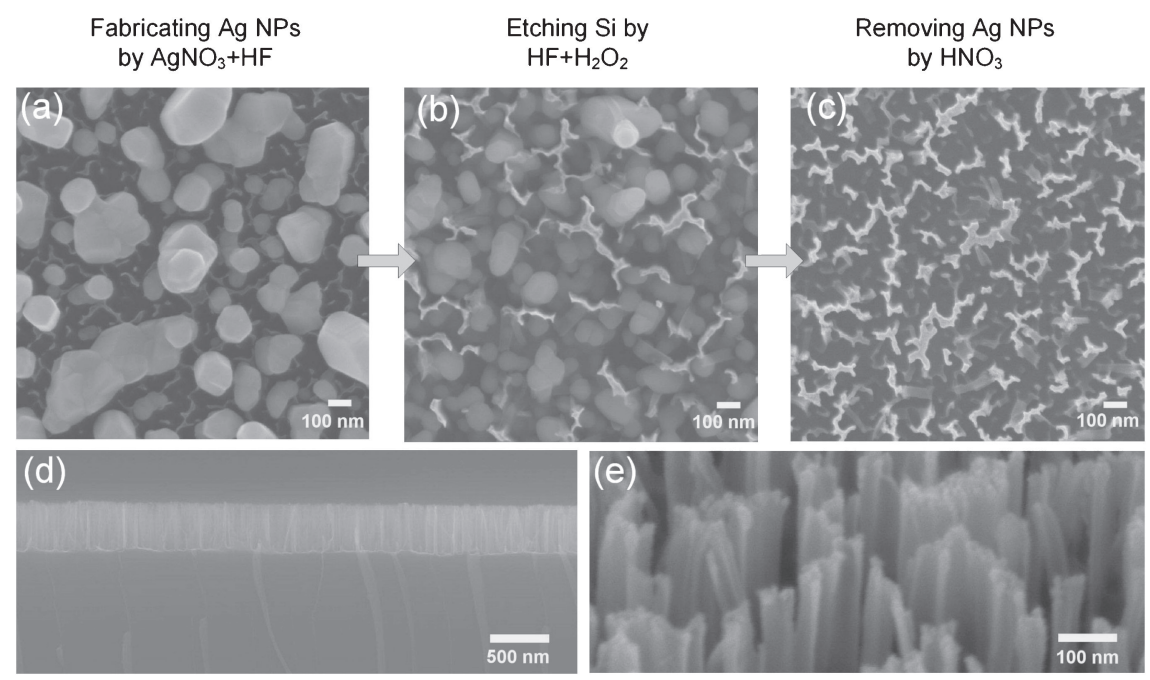

Fig. 1 SEM images of (a) $\mathrm{Ag}$ NPs reduced from $\mathrm{AgNO}_{3}$ onto a silicon wafer, (b) Ag NPs embedded in silicon nanowalls, (c) the silicon nanowalls obtained after removing the $\mathrm{Ag} \mathrm{NPs}$ with $\mathrm{HNO}_{3}$, and (d) a cross-section of the nanowalls after etching for 1 min; (e) an enlarged tilted view of the image in (d).

\section{Results and Discussions}

Figure 1(a) displays a representative SEM image of a silicon wafer surface presenting Ag NPs reduced from $0.02 \mathrm{M} \mathrm{AgNO}_{3}$ and $\mathrm{HF}$ solution. As the $\mathrm{AgNO}_{3}$ concentration increased, these Ag NPs aggregated into a dendrite block on the surface. Embedded Ag NPs were evident within the holes between the nanowalls after removing the nonadsorbed Ag NPs (most existed in the form of a dendrite block) through washing with DI water and then etching for $1 \mathrm{~min}$ in $\mathrm{HF} / \mathrm{H}_{2} \mathrm{O}_{2}$ solution (Fig. 1(b)). After using $\mathrm{HNO}_{3}$ solution to remove the Ag NPs, we obtained a fresh nanowall morphology lacking any NPs (Fig. 1(c)). Interestingly, the holes between the nanowalls did not correspond exactly to the distribution of Ag NPs, suggesting that the nanowall morphology was not defined by the NP shape or distribution alone. In fact, the nanowall morphology beneath the NPs was evident prior to the $\mathrm{HF} / \mathrm{H}_{2} \mathrm{O}_{2}$ etching step (Fig. 1(a)). We suspect that both the NPs and atomic-scale Ag species acted as catalysts to promote the etching of the underlying silicon. ${ }^{17)}$ Figure 1(d) displays the cross-section of the Si nanowall after etching for $1 \mathrm{~min}$. The uniform height indicates that the etching rate was nearly constant over the silicon surface. A tilted-view SEM image at higher magnification (Fig. 1(e)) also clearly reveals the nanowall morphology.

Figure 2 displays the variations in morphology obtained after varying both the $\mathrm{AgNO}_{3}$ concentration and the $\mathrm{H}_{2} \mathrm{O}_{2}$ / $\mathrm{HF}$ etching time. At a low $\mathrm{AgNO}_{3}$ concentration (0.001 M), nanoholes were formed initially. Upon extending the etching time, the surface roughness increased, implying that the etching process was determined by the $\mathrm{Ag}$ content and that an insufficient number of Ag NPs might cause a rough surface as a result of nonuniform distribution. On the other hand, as the concentration of $\mathrm{AgNO}_{3}$ increased, most the surface was covered with $\mathrm{Ag}$ species, thereby decreasing the surface filling ratio (defined as the surface ratio of $\mathrm{Si}$ to air). The nanowalls may have buckled at longer etching times because of the low surface filling ratio.
Because the Ag NPs obtained from the reduction process were large (Figs. 1(a) and 1(b)), we removed them and deposited smaller Ag NPs through a thermal evaporation process. First, we deposited Ag on a flat silicon wafer to determine the deposition duration required for nanogap formation. Figures 3(a)-(c) display top-view SEM images of the samples deposited on flat silicon wafers for 30, 60, and $300 \mathrm{~s}$, respectively. As the deposition time increased, the discontinuous islands aggregated into a continuous film (Fig. 3(c)). Figure $3 d$ presents the gap width on the 60-s sample mapped using ImageJ software; it reveals that many of the gaps were characterized by a distance of less than $10 \mathrm{~nm}$. These narrow gaps have been suggested to account for the formation of hot spots for SERS applications. ${ }^{18)}$ Accordingly, we deposited Ag NPs onto the Si nanowalls for 30 or $60 \mathrm{~s}$. Figures 4(a) and 4(b) display tilted-view SEM images of the samples $\left(\mathrm{AgNO}_{3}\right.$ concentration, $0.06 \mathrm{M}$; etching time, $5 \mathrm{~min}$; evaporation time, $60 \mathrm{~s}$ ) prepared before and after Ag decoration, respectively; in the latter, we observe discrete NPs on the tops and sides of the nanowalls. Moreover, Fig. 4(c) reveals that the Ag NPs were also deposited within the pores and on the bottom of the nanowalls, demonstrating that the nanowalls were $3 \mathrm{D}$ substrates that could support more NPs than could appear on the flat surface.

Next, we used R6G dye as a probe molecule to examine the enhancement in sensitivity of Raman spectra arising from SERS. We used the $\mathrm{C}-\mathrm{O}$ stretching signal of R6G at $611 \mathrm{~cm}^{-1}$ as the characteristic peak (the strongest one in the range $550-1800 \mathrm{~cm}^{-1}$ in this study) to calculate the $E F$ s of the SERS sensitivity. We evaluated the EFs using the equation $^{19)}$

$$
E F=\frac{I_{\mathrm{SER}}}{I_{\mathrm{REF}}} \times \frac{N_{\mathrm{REF}}}{N_{\mathrm{SER}}} \times \frac{P_{\mathrm{REF}}}{P_{\mathrm{SER}}} \times \frac{t_{\mathrm{REF}}}{t_{\mathrm{SER}}}
$$

where $I, N, P$, and $t$ are the Raman spectrum intensity, number of molecules, laser power, and exposure time, respectively; SER and REF represent the values from the $\mathrm{Ag} /$ nanowall samples and the reference sample of bare silicon, respec- 


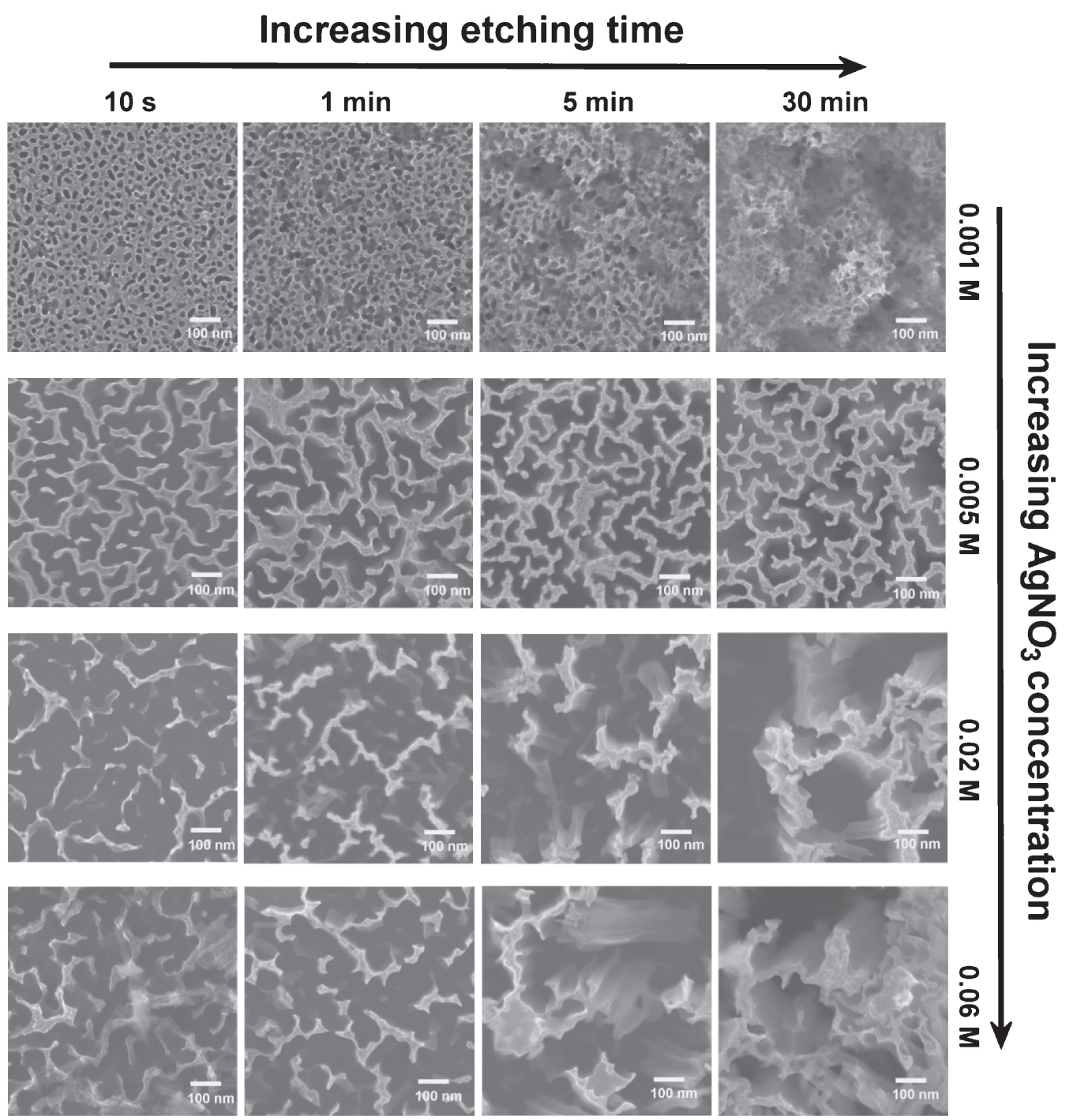

Fig. 2 Top-view SEM images of the samples obtained using various $\mathrm{AgNO}_{3}$ concentrations and $\mathrm{HF} / \mathrm{H}_{2} \mathrm{O}_{2}$ etching times.


Fig. 3 (a)-(c) SEM images of Ag NPs deposited on silicon wafers for (a) 30, (b) 60, and (c) 300 s. (d) Distance map of the gaps between $\mathrm{Ag}$ islands in the $60 \mathrm{~s}$ sample. 

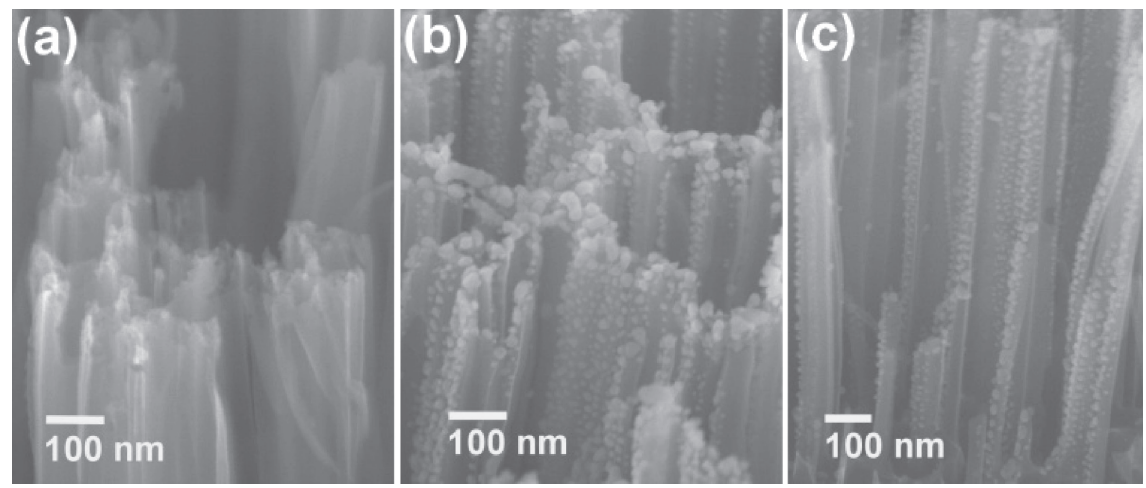

Fig. 4 (a), (b) Tilted-view SEM images of the silicon nanowalls decorated (a) before and (b) after Ag NPs. (c) SEM image of the nanowalls with Ag NPs deposited entirely along the sidewalls.
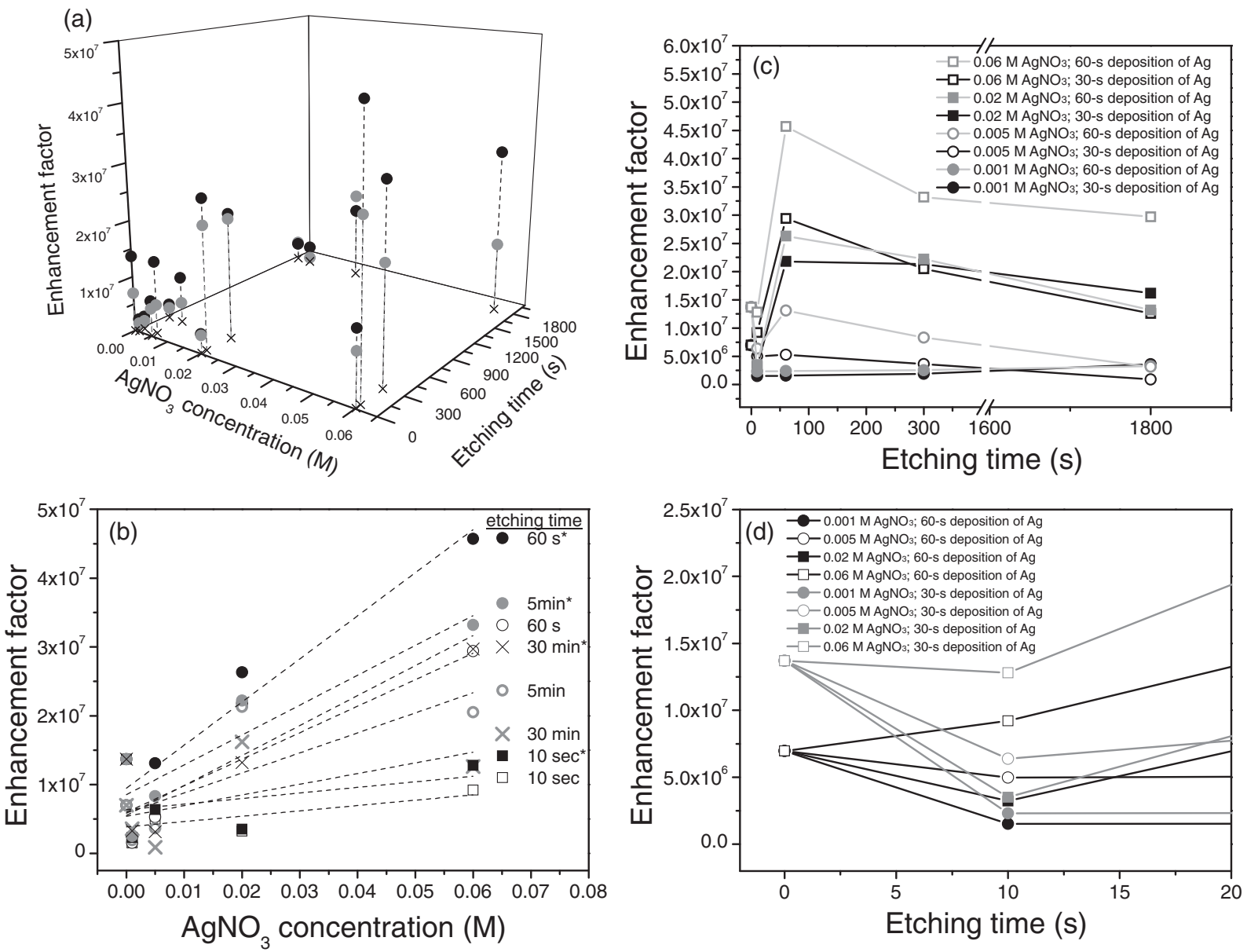

Fig. 5 (a) Distribution of $E F \mathrm{~s}$ for samples prepared at various $\mathrm{AgNO}_{3}$ concentrations and etching times. Black dots: $60 \mathrm{~s}$ deposition of $\mathrm{Ag}$; gray dots: $30 \mathrm{~s}$ deposition of $\mathrm{Ag}$. (b) Dependence of $E F$ on $\mathrm{AgNO}_{3}$ concentration. An asterisk represents a sample prepared with an evaporation time of $60 \mathrm{~s}$; the absence of an asterisk represents a $30 \mathrm{~s}$ evaporation time. (c) Dependence of $E F$ on $\mathrm{HF} / \mathrm{H}_{2} \mathrm{O}_{2}$ etching time; gray lines: 60 s deposition of Ag; black lines: $30 \mathrm{~s}$ deposition of Ag. (d) Enlarged view of Fig. (c) after a short etching time.

tively. Because each R6G solution had the same volume $(100 \mathrm{~mL})$, we used the ratio of the $\mathrm{R} 6 \mathrm{G}$ concentrations, instead of the ratio of the number of molecules $\left(N_{\mathrm{REF}} / N_{\mathrm{SER}}\right)$, to calculate the EFs. Figure 5(a) presents the $3 \mathrm{D} \mathrm{EF}$ distribution as a function of the $\mathrm{AgNO}_{3}$ concentration and the $\mathrm{HF} / \mathrm{H}_{2} \mathrm{O}_{2}$ etching time when using $10^{-7} \mathrm{M}$ R6G solution for measurement on the $\mathrm{Ag} /$ nanowall sample; we used a much higher concentration of R6G $\left(10^{-2} \mathrm{M}\right)$ for bare silicon as the reference because the Raman signal is very weak in the absence of silver. The samples decorated with the Ag NPs deposited for 30 and $60 \mathrm{~s}$ exhibited similar trends in terms of their sensitivities with respect to the $\mathrm{AgNO}_{3}$ concentration and $\mathrm{HF} / \mathrm{H}_{2} \mathrm{O}_{2}$ etching time; in general, the sensitivities of the $60 \mathrm{~s}$ samples were higher than those of the $30 \mathrm{~s}$ samples, presumably because of the shorter distance, which provided stronger hot spots for SERS, between their NPs. The $E F$ increased upon increasing the $\mathrm{AgNO}_{3}$ concentration (Fig. 5(b)), suggesting that a greater content of $\mathrm{AgNO}_{3}$ 


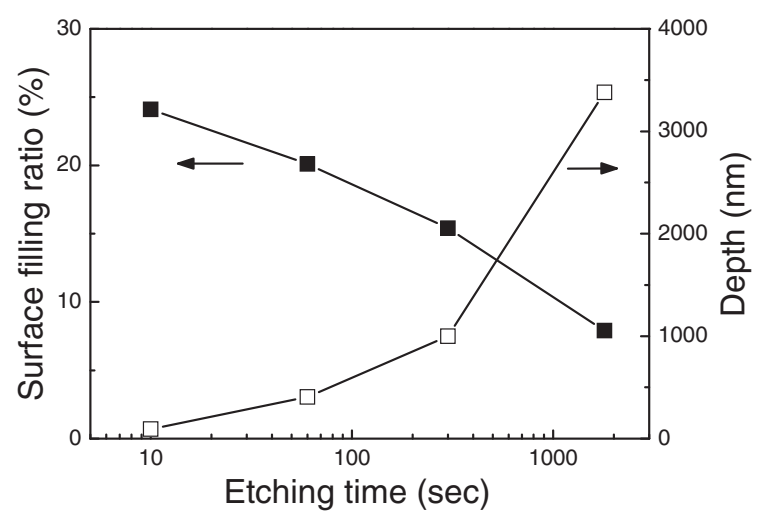

Fig. 6 Depth and surface filling ratio of nanowalls plotted with respect to the $\mathrm{HF} / \mathrm{H}_{2} \mathrm{O}_{2}$ etching time (logarithmic scale). These samples were prepared using $0.06 \mathrm{M} \mathrm{AgNO}_{3}$.

broadened the pores between the nanowalls and allowed the subsequently evaporated $\mathrm{Ag}$ atoms to deposit readily within them. On the other hand, the $E F$ reached its maximum value $\left(4.57 \times 10^{7}\right)$ upon increasing the $\mathrm{HF} / \mathrm{H}_{2} \mathrm{O}_{2}$ etching time to $60 \mathrm{~s}$ (Fig. 5(c)). This value is approximately three times higher than that of the system in which the Ag atoms were deposited onto a flat surface $\left(E F=1.37 \times 10^{7}\right)$; further increases in the etching time did not lead to higher $E F$ s. Figure 5(d) reveals that after a very short etching time (10 s) the $E F$ was even less than that obtained from the flat wafer, indicating that at the onset of etching the number of deposited Ag NPs on the rough surface was not higher than that on the flat surface. The existence of an optimal depth for SERS can be explained by considering the trade-off between the depth and the surface filling ratio. Figure 6 displays the typical variation in depth and surface filling ratio plotted with respect to the etching time. We measured the depth from the crosssectional SEM images, and obtained the surface filling ratio by analyzing the top-view SEM images with ImageJ software. We could control the depth with uniform heights, from several tens of nanometers to several micrometers, by varying the duration of etching with $\mathrm{HF} / \mathrm{H}_{2} \mathrm{O}_{2}$. The trade-off between depth and filling ratio determines the SERS $E F$. Typically, the $E F$ arises from hot spots between the NPs. Therefore, we ascribe the variations in SERS sensitivity to the different number of hot spots. For samples prepared with the shortest etching time $(10 \mathrm{~s})$, the rough surface decreased the particle packing density; accordingly, the EF decreased. On the other hand, at longer etching times (between 5 and $30 \mathrm{~min}$ ), although the sides of the nanowalls provided greater areas for deposition of $\mathrm{Ag} \mathrm{NPs}$, the surface filling ratios decreased as a result of buckling between the higher nanowalls (Fig. 2). Therefore, the overall number of NPs was not as high as that obtained at the optimal etching time (60 s); accordingly, we observed a lower SERS EF at longer etching times. Notably, in this study the optimal depth was only approximately $400 \mathrm{~nm}$ - for the sample prepared from $0.06 \mathrm{M} \mathrm{AgNO}_{3}$ and etched under the $\mathrm{HF} / \mathrm{H}_{2} \mathrm{O}_{2}$ solution for $1 \mathrm{~min}$; this depth, which is less than that obtained typically through the MACE process, should benefit the development of flexible or thin-film SERS devices. SERS with higher sensitivity would be expected if the high surface filling ratio could be maintained for deeper nanowalls.

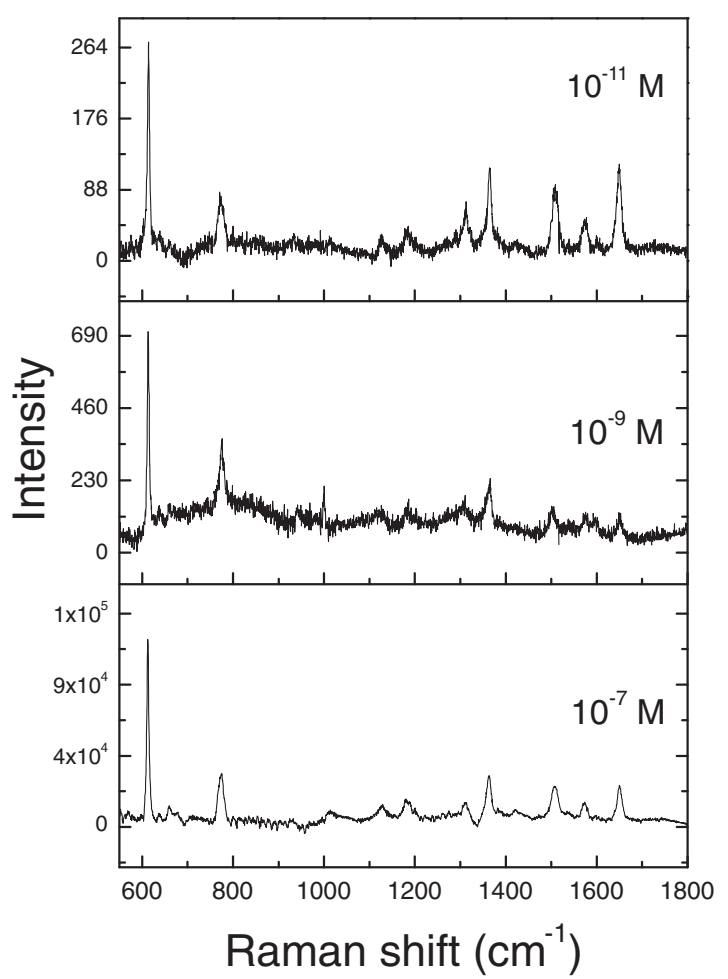

Fig. 7 SERS spectra of R6G collected on the 400-nm-thick nanowall sample at various $\mathrm{R} 6 \mathrm{G}$ concentrations.

The high sensitivity of 400-nm-deep nanowalls could be used to detect lower concentrations of the dye R6G. Figure 7 presents Raman spectra recorded with $\mathrm{R} 6 \mathrm{G}$ concentrations ranging from $10^{-7}$ to $10^{-11} \mathrm{M}$. The characteristic signals of R6G were still clearly identifiable at $10^{-11} \mathrm{M}$, but with lower Raman intensity. Interestingly, the Raman intensity did not decrease proportionally upon decreasing the R6G concentration, such that we obtained a higher $E F$ at lower concentration $\left(E F=1.1 \times 10^{9}\right.$ at $\left.10^{-11} \mathrm{M}\right)$. We suspect that the opportunities for contact between the hot spots and R6G molecules were higher in the more-dilute R6G solutions, but the greater number of molecules in the denser solutions were presumably blocked by other molecules adhered to the nanowall surfaces, thereby resulting in lower $E F \mathrm{~s}$.

\section{Conclusion}

We have used metal-assisted chemical etching and thermal evaporation processes to prepare thin silicon nanowalls decorated with Ag NPs to increase the $E F$ for the detection of R6G molecules on silicon wafers. SEM images indicated that we could obtain thin silicon nanowalls of high uniformity through MACE in this study by removing nonadsorbed $\mathrm{Ag} \mathrm{NPs}$ prior to $\mathrm{HF} / \mathrm{H}_{2} \mathrm{O}_{2}$ etching, allowing tinier $\mathrm{Ag}$ NPs to be evaporated onto the tops and sides of the nanowalls. By controlling several experimental parametersthe $\mathrm{AgNO}_{3}$ concentration, the $\mathrm{HF} / \mathrm{H}_{2} \mathrm{O}_{2}$ etching time, and the duration of thermal evaporation-we found that both the surface filling ratio and the etching depth of the nanowalls could be adjusted for optimal SERS performance. Increasing the $\mathrm{AgNO}_{3}$ concentration could improve the etching uniformity over the surface, but higher nanowalls tended to buckle together after longer etching times, thereby decreasing 
the surface filling ratio and leading to lower $E F$ s. For a $10^{-7} \mathrm{M}$ solution of the dye R6G, the 400-nm-deep nanowalls could be applied to increase the SERS EF up to $4.57 \times 10^{7}$, approximately three times higher than that of the NPs on the flat surface. The Raman spectrum identified R6G clearly even when we had decreased its concentration to $10^{-11} \mathrm{M}$. This readily processed, highly sensitive, thin nanowall substrate appears to be an attractive material for further research into low-cost, large-area SERS sensors on flexible substrates.

\section{Acknowledgments}

We thank the National Science Council, Taiwan, Republic of China, for financial support (contract NSC 101-2628-E239-002-MY3). We also thank Professor Yi-Sheng Lai for help with the thermal evaporation process, and the Center for Micro/Nano Science and Technology at National Cheng Kung University for Raman measurement.

\section{REFERENCES}

1) B. Sharma, R. R. Frontiera, A. Henry, E. Ringe and R. P. Van Duyne: Mater. Today 15 (2012) 16-25.

2) H. Ko, S. Singamaneni and V. V. Tsukruk: Small 4 (2008) 1576-1599.

3) G. Cao: Nanostructures and Nanomaterials: Synthesis, Properties, and
Applications, (Imperial College Press, London, 2004) pp. 51-109.

4) M. J. Natan: Faraday Discuss. 132 (2006) 321-328.

5) S. Chattopadhyay, H. C. Lo, C. H. Hsu, L. C. Chen and K. H. Chen: Chem. Mater. 17 (2005) 553-559.

6) K. Sun, G. Meng, Q. Huang, X. Zhao, C. Zhu, Z. Huang, Y. Qian, X. Wang and X. Hu: J. Mater. Chem. C 1 (2013) 5015-5022.

7) X. Hu, G. Meng, Q. Huang, W. Xu, F. Han, K. Sun, Q. Xu and Z. Wang: Nanotechnology 23 (2012) 385705.

8) B. Zhang, H. Wang, L. Lu, K. Ai, G. Zhang and X. Cheng: Adv. Funct. Mater. 18 (2008) 2348-2355.

9) M. Peng, J. Gao, P. Zhang, Y. Li, X. Sun and S. T. Lee: Chem. Mater. 23 (2011) 3296-3301.

10) M. Peng, H. Xu and M. Shao: Appl. Phys. Lett. 104 (2014) 193103.

11) S. Siddhanta, V. Thakur, C. Narayana and S. M. Shivaprasad: ACS Appl. Mater. Interfaces 4 (2012) 5807-5812.

12) M. L. Seol, S. J. Choi, D. J. Baek, T. J. Park, J. H. Ahn, S. Y. Lee and Y. K. Choi: Nanotechnology 23 (2012) 095301.

13) H. Ko and V. V. Tsukruk: Small 4 (2008) 1980-1984.

14) Z. Huang, N. Geyer, P. Werner, J. de Boor and U. Gösele: Adv. Mater. 23 (2011) 285-308.

15) M. L. Zhang, K. Q. Peng, X. Fan, J. S. Jie, R. Q. Zhang, S. T. Lee and N. B. Wong: J. Phys. Chem. C 112 (2008) 4444-4450.

16) M. Ohring: Materials Science of Thin Films, (Academic Press, London, 2002) pp. 95-144.

17) S. C. Tseng, H. L. Chen, C. C. Yu, Y. S. Lai and H. W. Liu: Energy Environ. Sci. 4 (2011) 5020-5027.

18) H. H. Wang, C. Y. Liu, S. B. Wu, N. W. Liu, C. Y. Peng, T. H. Chan, C. F. Hsu, J. K. Wang and Y. L. Wang: Adv. Mater. 18 (2006) 491-495.

19) M. Zamuner, D. Talaga, F. Deiss, V. Guieu, A. Kuhn, P. Ugo and N. Sojic: Adv. Funct. Mater. 19 (2009) 3129-3135. 